\title{
SINGULARITIES OF GENERALIZED PARTON DISTRIBUTIONS
}

\author{
ANATOLY V. RADYUSHKIN* \\ Physics Department, Old Dominion University, \\ Norfolk, VA 23529, USA \\ and \\ Thomas Jefferson National Accelerator Facility, \\ Newport News, VA 23606, USA \\ radyush@jlab.org
}

\begin{abstract}
We discuss recent developments in building models for GPDs that are based on the formalism of double distributions (DDs). A special attention is given to a careful analysis of the singularity structure of DDs. The DD formalism is applied to construction of a model GPDs with a singular Regge behavior. Within the developed DD-based approach, we discuss the structure of GPD sum rules. It is shown that separation of DDs into the so-called "plus" part and the $D$-term part may be treated as a renormalization procedure for the GPD sum rules. This approach is compared with an alternative prescription based on analytic regularization.
\end{abstract}

Keywords: Generalized parton distributions; Regge behavior; sum rules; analytic regularization.

PACS numbers: 11.10.-z, 12.38.-t, 13.60.Fz

\section{Introductory Remarks}

The basic role played by the generalized parton distributions (GPDs) ${ }^{1-7}$ is to access the fundamental physics related to the structure of hadrons. In the absence of solutions of QCD equations of motion, the standard approach is the description of hadron structure in terms of phenomenological functions, such as form factors, parton densities, and distribution amplitudes. These "old" functions were used for a long a time before the GPDs were proposed. The "new" functions, GPDs (for reviews, see Refs. [8-12]), are hybrids of the "old" functions. Furthermore, the "old" functions are limiting cases of the "new" ones.

The relation of GPDs to more simple "old" functions is an essential element in constructing realistic models of GPDs. One of the most restrictive constraints is imposed by the formula ${ }^{2}$ relating GPDs to the usual parton densities, which may be treated as "forward" limit of GPDs. A nontrivial observation here is that GPDs contain contributions which are "invisible" in the forward limit, such as the

*Also at Bogoliubov Laboratory of Theoretical Physics, JINR, 141980 Dubna, Russian Federation. 
$D$-term. ${ }^{13}$ In addition to the requirements of reproducing "old" functions in specified limits ("reduction relations"), such models should satisfy other constraints, such as polynomiality, ${ }^{8}$ and correspondence with the Regge behavior of usual parton densities in the region of small parton momenta.

The polynomiality constraint is highly nontrivial, but it is automatically satisfied if GPDs are built from so-called "double distributions" . 1, 4,6 However, imposing on DDs the constraints dictated by correspondence with the Regge behavior one faces rather singular functions, and this raises a lot of questions related to the singularity structure of GPDs in general.

An overview of the basic ideas of the GPD theory was given in Ref. [14], which may be treated as an introduction to this work. The goal of the present paper is to describe a recent development ${ }^{15}$ in modeling GPDs, based on their formulation in terms of double distributions, with emphasis on careful disentangling their singularity structure.

In particular, we address a specific problem of building model GPDs with the Regge behavior. The model described in Section 2, provides a particular example of singularities that one may encounter in GPD construction. It also gives a nontrivial example of a situation when the part of the $D$-term (that is formally invisible in the forward limit), comes from a term generated by the correspondence with the usual ("forward") parton densities. As shown in recent papers, ${ }^{16-20}$ the $D$ term also appears as a subtraction constant in dispersion sum rules for GPDs. In Section 3 , we study these sum rules within the DD formalism used in previous sections, in particular, we show that separation of DDs into the so-called "plus" part and the $D$-term part may be treated as a renormalization procedure for the GPD sum rules. In Section 4, we compare it to the alternative prescription based on analytic regularization used in Refs. [18, 21, 22]. Then we formulate our conclusions.

\section{Modeling GPDs}

Preliminaries. The general idea of extracting GPDs from experiments is to build some models for GPDs, and fix the parameters of such models by comparing their predictions with experimental data. There are two approaches used to model GPDs. One is based on a direct calculation of parton distributions in specific dynamical models, such as bag model, ${ }^{23}$ chiral soliton model, ${ }^{24}$ light-cone formalism, ${ }^{25}$ etc. Another approach ${ }^{26-28}$ is a phenomenological construction based on reduction formulas relating GPDs to usual parton densities $f(x), \Delta f(x)$ and form factors $F_{1}(t), F_{2}(t), G_{A}(t), G_{P}(t)$. The most convenient way to construct such models is to start with double distributions $f(\beta, \alpha ; t)$.

We will concentrate on the limiting case $t=0$. One can generate GPDs $H(x, \xi)$ for different values of the skewness parameter $\xi$ from universal $\xi$-independent double distributions $f(\beta, \alpha)$. For a spin-0 target, the single-DD representation ${ }^{29}$ gives

$$
\frac{H(x, \xi)}{x}=\int_{\Omega} f(\beta, \alpha) \delta(x-\beta-\xi \alpha) d \beta d \alpha .
$$


In the forward limit $\xi=0, \operatorname{GPD} H(x, \xi)$ converts into the usual parton distribution $f(x) / x$. Using DDs, we may write

$$
f(x)=x \int_{-1+|x|}^{1-|x|} f(x, \alpha) d \alpha .
$$

Thus, the forward distributions $f(x)$ are obtained by integrating DDs over vertical lines $\beta=x$ in the $(\beta, \alpha)$ plane. For nonzero $\xi$, GPDs are obtained from DDs through integrating them along the lines $\beta=x-\xi \alpha$ having $1 / \xi$ slope. The reduction formula (2) suggests the factorized DD Ansatz

$$
f(\beta, \alpha)=h(\beta, \alpha) f(\beta) / \beta,
$$

where $f(\beta)$ is the forward distribution, while $h(\beta, \alpha)$ determines DD profile in the $\alpha$ direction and satisfies the normalization condition

$$
\int_{-1+|\beta|}^{1-|\beta|} h(\beta, \alpha) d \alpha=1
$$

The profile function should be symmetric with respect to $\alpha \rightarrow-\alpha$ because DDs $f(\beta, \alpha)$ are even in $\alpha{ }^{27,30}$ For a fixed $\beta$, the function $h(\beta, \alpha)$ describes how the longitudinal momentum transfer $r^{+}$is shared between the two partons (see, e.g. Ref. [14]). Hence, it is natural to expect that the shape of $h(\beta, \alpha)$ should look like a symmetric meson distribution amplitude (DA) $\varphi(\alpha)$. Since DDs have the support restricted by $|\alpha| \leq 1-|\beta|$, to get a more complete analogy with DAs, it makes sense to rescale $\alpha$ as $\alpha=(1-|\beta|) \gamma$ introducing the variable $\gamma$ with $\beta$-independent limits: $-1 \leq \gamma \leq 1$. The simplest model is to assume that the $\gamma$-profile is a universal function $g(\gamma)$ for all $\beta$. Possible simple choices for $g(\gamma)$ may be $\delta(\gamma)$ (no spread in $\gamma$-direction), $\frac{3}{4}\left(1-\gamma^{2}\right)$ (characteristic shape for asymptotic limit of nonsinglet quark distribution amplitudes), $\frac{15}{16}\left(1-\gamma^{2}\right)^{2}$ (asymptotic shape of gluon distribution amplitudes), etc. In the variables $\beta, \alpha$, these models can be treated as specific cases of the general profile function

$$
h^{(N)}(\beta, \alpha)=\frac{\Gamma(2 N+2)}{2^{2 N+1} \Gamma^{2}(N+1)} \frac{\left[(1-|\beta|)^{2}-\alpha^{2}\right]^{N}}{(1-|\beta|)^{2 N+1}},
$$

whose width is governed by the parameter $N$.

Integrating $\mathrm{DD} f(\beta, \alpha)$ with respect to $\beta$ gives the $D$-term ${ }^{13}$

$$
D(\alpha)=\alpha \int_{-1+|\alpha|}^{1-|\alpha|} f(\beta, \alpha) d \beta,
$$

and one may write DD as a sum

$$
f(\beta, \alpha)=[f(\beta, \alpha)]_{+}+\delta(\beta) \frac{D(\alpha)}{\alpha}
$$

of its $D$-term part $\delta(\beta) D(\alpha) / \alpha$ and the "plus" part

$$
[f(\beta, \alpha)]_{+} \equiv f(\beta, \alpha)-\delta(\beta) \int_{-1+|\alpha|}^{1-|\alpha|} f(\gamma, \alpha) d \gamma .
$$



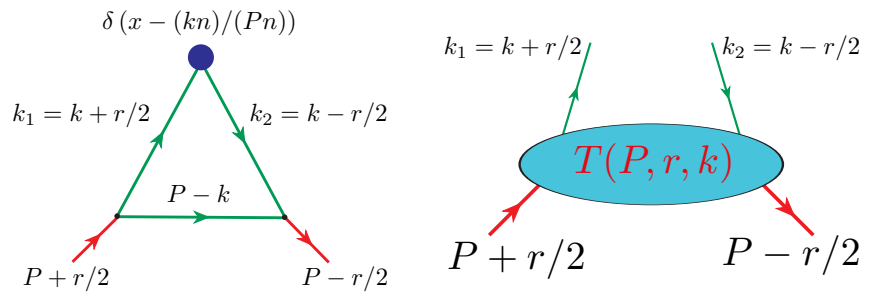

Fig. 1. Left: Triangle diagram model for GPD. Right: Hadron-quark scattering amplitude.

For the GPD $H(x, \xi)$, the "DD++ D" separation corresponds to the representation

$$
H(x, \xi) \equiv H_{+}(x, \xi)+D(x / \xi)
$$

Diagrammatic model for GPDs with implanted Regge behavior. Assumptions used in the factorized DD Ansatz are based on the experience with calculating DDs for triangle diagrams ${ }^{6}$ and form factors in the light-front formalism models with power-law dependence of the wave function on transverse momentum ${ }^{31}$ (see also Ref. [32]). The simplest triangle diagram (see Fig. 1, left) in a purely scalar model may be used as an example of a model for GPD

$$
H(x, \xi) \sim \int \frac{d^{4} k \delta(x-(k n) /(P n))}{\left(m_{1}^{2}-k_{1}^{2}\right)\left(m_{2}^{2}-k_{2}^{2}\right)\left(m_{3}^{2}-(P-k)^{2}\right)} .
$$

Though the $\xi$-dependence is not immediately visible here, it appears after integration over $k$ through the $(r n) / 2(P n)$ ratio. The $\mathrm{DD} F(\beta, \alpha)$ generated by this diagram is just a constant, see Ref. [6], which corresponds to a flat $N=0$ profile $h^{(0)}(\beta, \alpha) \sim 1 /(1-\beta)$ and $f(\beta) \sim 1-\beta$ forward distribution.

The calculation $^{31}$ of overlap integrals for light-front wave functions with a powerlaw behavior $\psi\left(x, k_{\perp}\right) \sim 1 /\left(k_{\perp}^{2}\right)^{1+\kappa}$ resulted in expressions equivalent to using DDs with $N=\kappa$ profile in Eq. (5) and forward distributions behaving like $(1-\beta)^{2 \kappa+1}$. The same profile arises ${ }^{31}$ if one differentiates a scalar triangle diagram $\kappa$ times with respect to masses (squared) of each active quark, i.e. substitutes

$$
\frac{1}{\left(m_{1}^{2}-k_{1}^{2}\right)\left(m_{2}^{2}-k_{2}^{2}\right)} \rightarrow \frac{1}{\left(m_{1}^{2}-k_{1}^{2}\right)^{1+\kappa}\left(m_{2}^{2}-k_{2}^{2}\right)^{1+\kappa}} .
$$

The triangle diagrams, however, do not generate the Regge $f(\beta) \sim 1 / \beta^{a}$ behavior for small $\beta$. The latter may be obtained, in particular, by infinite summation of higher-order $t$-channel ladder diagrams (see, e.g., Ref. [33]). A simpler way was proposed in Ref. [34], where the spectator propagator was substituted by a partonhadron scattering amplitude $T(P, r, k)$ (see Fig. 1, right) ) written in the dispersion relation representation with subtraction

$$
T(P, r, k) \rightarrow T\left((P-k)^{2}\right)=T_{0}+\int_{0}^{\infty} d \sigma \rho(\sigma)\left\{\frac{1}{\sigma-(P-k)^{2}}-\frac{1}{\sigma}\right\} .
$$


The spectral function $\rho(\sigma)$ here should be adjusted to produce a desired Regge-type behavior with respect to $s=(P-k)^{2}$.

The model of Ref. [34] assumes spin-1/2 quarks. It was argued that the Dirac structure of the hadron-parton scattering amplitude in this case should be given by $\not k$, which provides EM gauge invariance of the DVCS amplitude. Thus, the model scattering amplitude has the structure

$$
\frac{\not k T\left((P-k)^{2}\right.}{\left(m_{1}^{2}-k_{1}^{2}\right)^{N_{1}+1}\left(m_{2}^{2}-k_{2}^{2}\right)^{N_{2}+1}} .
$$

Treating two quarks on equal footing, $m_{1}=m_{2}$, gives the following model GPD

$$
\begin{aligned}
H(x, \xi)= & \frac{1}{\pi^{2}} \frac{N_{1} ! N_{2} !}{\left(N_{1}+N_{2}\right) !} \int \frac{(k n)}{(P n)} \frac{d^{4} k \delta(x-(k n) /(P n))}{\left[m^{2}-(k+r)^{2}\right]^{N_{1}+1}\left[m^{2}-(k-r)^{2}\right]^{N_{2}+1}} \\
& \times\left[T_{0}+\int_{0}^{\infty} d \sigma \rho(\sigma)\left\{\frac{1}{\sigma-(P-k)^{2}}-\frac{1}{\sigma}\right\}\right] .
\end{aligned}
$$

The overall factors were introduced here for future convenience. The $T_{0}$ subtraction term gives the $D$-term-type contribution

$$
D_{0}(x / \xi)=\frac{T_{0}}{2^{N_{1}+N_{2}}\left(N_{1}+N_{2}\right)}\left(\frac{x}{|\xi|}\right)\left(1-\frac{x}{\xi}\right)^{N_{1}}\left(1+\frac{x}{\xi}\right)^{N_{2}} \theta\left(\left|\frac{x}{\xi}\right|<1\right) .
$$

Let us consider now less trivial terms generated by the dispersion integral.

The model and $\boldsymbol{D} D$ representation. For equal $N_{1}=N_{2}=N$, we obtain

$$
\begin{aligned}
H(x, \xi) & =\frac{x}{2^{2 N+1}} \int_{0}^{\infty} d \sigma \rho(\sigma) \int_{0}^{1} d \beta \int_{-1+\beta}^{1-\beta} d \alpha \frac{\left[(1-\beta)^{2}-\alpha^{2}\right]^{N}}{\left(\beta \sigma+(1-\beta) m^{2}\right)^{2 N+1}} \\
& \times\left\{\delta(x-\beta-\alpha \xi)-\frac{\delta(x-\alpha \xi /(1-\beta))}{(1-\beta)^{2}}\right\} .
\end{aligned}
$$

Taking $\xi=0$, one obtains the usual (forward) parton distributions:

$$
f(x)=\frac{(N !)^{2}}{(2 N+1) !} x(1-x)^{2 N+1} \int_{0}^{\infty} \frac{d \sigma \rho(\sigma)}{\left(x \sigma+(1-x) m^{2}\right)^{2 N+1}} .
$$

Now, one can substitute the $\sigma$-integral through forward distribution to get

$$
\begin{aligned}
H(x, \xi)= & \frac{x}{2^{2 N+1}} \frac{(2 N+1) !}{(N !)^{2}} \int_{0}^{1} d \beta \int_{-1+\beta}^{1-\beta} d \alpha \frac{\left[(1-\beta)^{2}-\alpha^{2}\right]^{N}}{(1-\beta)^{2 N+1}} \frac{f(\beta)}{\beta} \\
& \times\left\{\delta(x-\beta-\alpha \xi)-\frac{\delta(x-\alpha \xi /(1-\beta))}{(1-\beta)^{2}}\right\} .
\end{aligned}
$$

In this expression it is easy to recognize the normalized profile function $h_{N}(\beta, \alpha)$ of Eq. (5). Thus, we can rewrite Eq. (18) as

$$
\begin{aligned}
\frac{H(x, \xi)}{x}= & \int_{0}^{1} d \beta \int_{-1+\beta}^{1-\beta} d \alpha \frac{f(\beta)}{\beta} h_{N}(\beta, \alpha) \\
& \times\left\{\delta(x-\beta-\alpha \xi)-\frac{\delta(x-\alpha \xi /(1-\beta))}{(1-\beta)^{2}}\right\} .
\end{aligned}
$$


The first term here coincides with the factorized DD Ansatz for GPD $H(x, \xi) / x$ in which it is reconstructed from its forward limit $f(x) / x$. The relevant double distribution is given by $f(\beta, \alpha)=h_{N}(\beta, \alpha) f(\beta) / \beta$. Changing $\alpha /(1-\beta) \rightarrow \alpha$ in the second delta function, we find that the total contribution may be written as

$$
\frac{H(x, \xi)}{x}=\int_{0}^{1} d \beta \int_{-1}^{1} d \alpha \delta(x-\beta-\alpha \xi)\left\{f(\beta, \alpha)-\delta(\beta) h(0, \alpha) \int_{0}^{1} d \gamma \frac{f(\gamma)}{\gamma(1-\gamma)^{2}}\right\}
$$

Thus, the model of Ref. [34] corresponds to the single-DD representation. ${ }^{29}$ Also, the first term in (20) has the structure of the factorized DD Ansatz (3).

Results for GPDs. For the model forward distribution $f_{a}(\beta)=(1-\beta)^{3} / \beta^{a}$ and the $N=1$ profile function $h^{(1)}(\beta, \alpha)$ of Eq. (5) we obtain, for $x>\xi$ :

$$
\left.H(x, \xi)\right|_{x>\xi}=\frac{3}{4} \frac{x}{\xi} \int_{\beta_{1}}^{\beta_{2}} \frac{d \beta}{\beta^{a+1}}\left\{(1-\beta)^{2}-\left(\frac{x-\beta}{\xi}\right)^{2}\right\} .
$$

At the border point $x=\xi$, one gets the $\left[(1-\beta)^{2}-(1-\beta / \xi)^{2}\right] \sim \beta$ factor from the profile function, which changes the strength of singularity for $\beta=0$. As a a consequence of using a profile function that linearly vanishes at the sides of the support rhombus, the integral over $\beta$ converges as far as $a<1$. In its turn, the $N=1$ profile is generated by the assumed $1 /\left(k_{1}^{2} k_{2}^{2}\right)^{2}$ dependence of the $k$-integrand for large parton virtualities. If one takes the $N=0$ profile, the factor in the curly brackets should be substituted by $1 /(1-\beta)$, and the integral producing $H(\xi, \xi)$ diverges. Turning now to the $|x|<\xi$ region we get, for the $N=1$ profile:

$$
\begin{aligned}
\left.H(x, \xi)\right|_{|x|<\xi}= & \frac{3}{4} \frac{x}{\xi}\left[\frac{1}{\xi^{2}} \int_{0}^{\beta_{2}} \frac{d \beta}{\beta^{a}}(2 x-\beta)+\int_{0}^{\beta_{2}} \frac{d \beta}{\beta^{a}}\left\{1-\frac{x^{2}}{\xi^{2}(1-\beta)^{2}}\right\}(\beta-2)\right. \\
& \left.-\int_{\beta_{2}}^{1-|x| / \xi} \frac{d \beta}{\beta^{a+1}}\left\{1-\frac{x^{2}}{\xi^{2}(1-\beta)^{2}}\right\}\right]
\end{aligned}
$$

Note that as far as $|x|$ is strictly less than $\xi$, the profile function does not vanish at the singularity point $\beta=0$. The mechanism of softening singularity to $1 / \beta^{a}$ strength is now provided by the $1 / \sigma$ subtraction term of the original dispersion relation. To get a model for singlet GPDs, one should take the antisymmetric combination $H_{S}(x, \xi)=H(x, \xi)-H(-x, \xi)$. The resulting GPDs are shown in Fig. 2, left. For positive $x$, they are peaking at $x=\xi$. The functions $H_{S}(x, \xi)$ in this model are continuous at $x= \pm \xi$, but the derivative $d H_{S}(x, \xi) / d x$ is discontinuous at these points. In a similar way, one can calculate model GPDs for the $N=2$ profile. The resulting GPDs are shown in Fig. 2, right. For positive $x$, they are peaking at points close to $x=\xi$. In the model with $N=2$ profile, both the functions $H_{S}(x, \xi)$ and their derivatives $d H_{S}(x, \xi) / d x$ are continuous at $x= \pm \xi$. 


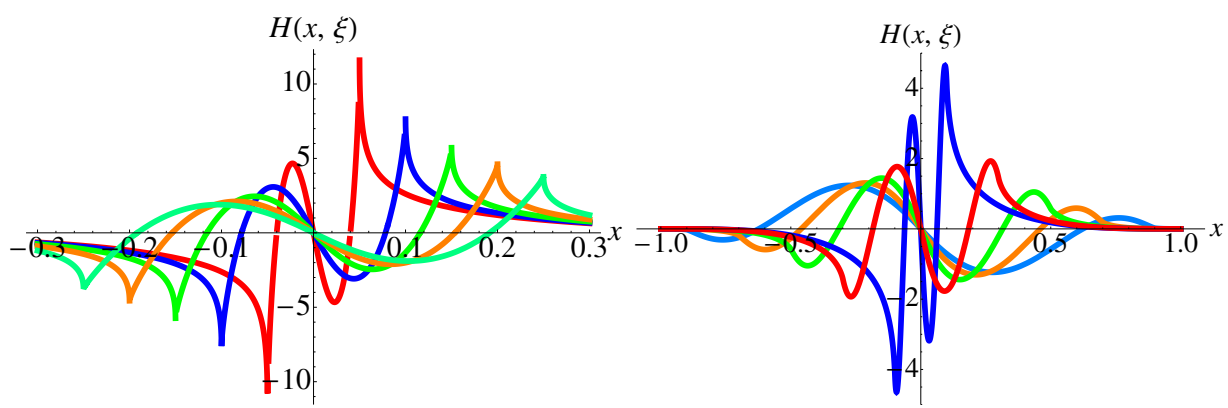

Fig. 2. Model singlet GPD $H_{S}(x, \xi)$ with $N=1$ (left) and $N=2$ (right) DD profile for $a=0.5$ and $\xi=0.05,0.1,0.15,0.2,0.25$.

Results for D-term. In Eq. (20), we deal with the regularized double distribution

$$
f^{\mathrm{reg}}(\beta, \alpha)=f(\beta, \alpha)-\delta(\beta) h(0, \alpha) \int_{0}^{1} d \gamma \frac{f(\gamma)}{\gamma(1-\gamma)^{2}} .
$$

However, the subtraction term in $f^{\mathrm{reg}}(\beta, \alpha)$ does not coincide with that in the definition (8) of $f_{+}(\beta, \alpha)$. Their difference induces the $D$-term

$$
D(\alpha)=2 \alpha \int_{0}^{1} d \beta \frac{f(\beta)}{\beta}\left\{h(\beta, \alpha)-\frac{h(0, \alpha)}{(1-\beta)^{2}}\right\} .
$$

Taking the model forward distribution $f(\beta)=(1-\beta)^{3} / \beta^{a}$, one can get explicit expressions for the $D$-term in case of $N=1$ and $N=2$ profile functions. As one can see in Fig. 3, the two curves are rather close to each other.

It is also interesting to look at another ingredient of GPD $H(x, \xi)$ : the term $H_{+}(x, \xi)$ obtained from the "plus" part $[f(\beta, \alpha)]_{+}$of DD. Formally, it corresponds to the difference between GPD $H(x, \xi)$ and $D$-term $D(x / \xi)$. The shape of this difference for $\xi=0.5$ is shown in Fig. 3, right. Note that, despite the fact that the forward distribution in this model is positive, there is a region, where the contribution to $H(x, \xi)$ coming from $[f(\beta, \alpha)]_{+}$is negative. This is due to the $\delta(\beta)$ subtraction term
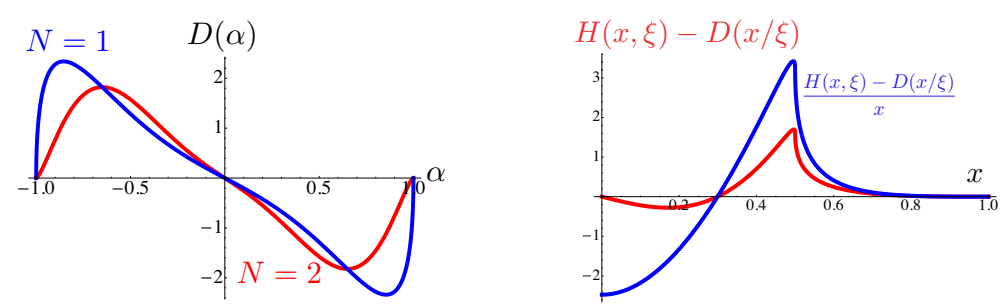

Fig. 3. Left: The $D$-terms in $N=1$ and $N=2$ profile models for $a=0.5$. Right: Difference between GPD $H(x, \xi)$ and $D$-term $D(x / \xi)$ in the case of the $N=1$ profile for $\xi=0.5$ and positive $x$. The same function divided by $x$ is also shown. 
contained in $[f(\beta, \alpha)]_{+}$. Also shown is the ratio $H_{+}(x, \xi) / x$. Looking at the figure, one may suspect that the $x$-integral of $H_{+}(x, \xi) / x$ vanishes. In the next section, we show that this, indeed, is the case.

\section{GPD Sum Rules}

Sum rules. The D-term determines the subtraction constant in the dispersion relation for the DVCS amplitude. ${ }^{16-20}$ In particular, it was shown ${ }^{17}$ that the original expression for the real part of the DVCS amplitude involving $H(x, \xi)$, and the dispersion integral involving $H(x, x)$ differ by a constant $\Delta$ given by the integral of the $D$-term function $D(\alpha)$ :

$$
P \int_{-1}^{1} \frac{H(x, x)-H(x, \xi)}{x-\xi} d x=\Delta \equiv \int_{-1}^{1} \frac{D(\alpha)}{1-\alpha} d \alpha .
$$

Here, $P$ denotes the principal value prescription. In Ref. [17], this relation was derived using polynomiality properties of GPDs.

Taking $\xi=0$, one formally arrives at the sum rule

$$
\int_{-1}^{1} \frac{H(x, x)-H(x, 0)}{x} d x=\int_{-1}^{1} \frac{D(\alpha)}{1-\alpha} d \alpha .
$$

Since both $H(x, 0) / x$ and $H(x, x) / x$ are even functions of $x$, their singularities for $x=0$ cannot be regularized by the principle value prescription. Moreover, there are no indications that singularities of these two functions cancel each other. On the contrary, as emphasized in Ref. [35], there are arguments that the ratio $H(x, x) / H(x, 0)$ does not tend to 1 for small $x$.

"Plus $+D$ " decomposition. To proceed, we start with the expression (1) producing GPDs from DDs, and the decomposition (8) of DD into the "plus" part $[f(\beta, \alpha)]_{+}$and the $D$-term part $\delta(\beta) D(\alpha) / \alpha$. Correspondingly, we split GPD into the part coming from the "plus" part of DD

$$
\frac{H_{+}(x, \xi)}{x} \equiv \int_{\Omega} f(\beta, \alpha)[\delta(x-\beta-\xi \alpha)-\delta(x-\xi \alpha)] d \beta d \alpha
$$

and that generated by the $D$-term

$$
\frac{H_{D}(x, \xi)}{x} \equiv \int_{-1}^{1} \frac{D(\alpha)}{\alpha} \delta(x-\xi \alpha) d \alpha
$$

An important relation

$$
\frac{H_{D}(x, 0)}{x}=\delta(x) \int_{-1}^{1} \frac{D(\alpha)}{\alpha} d \alpha
$$

is obtained by taking $\xi=0$. Eq. (27) also gives

$$
\frac{H_{D}(x, x)}{x}=\delta(x) \int_{-1}^{1} \frac{D(\alpha)}{\alpha(1-\alpha)} d \alpha .
$$

Note that both $H_{D}(x, 0) / x$ and $H_{D}(x, x) / x$ are proportional to $\delta(x)$, with the coefficients given by integrals of $D(\alpha)$. This means that, unlike the functions $H(x, 0)$ 
and $H(x, x)$, which, for $x \neq 0$, are insensitive to changes of $D(\alpha)$ in the $\delta(\beta) D(\alpha) / \alpha$ term, the (mathematical) distributions $H(x, 0) / x$ and $H(x, x) / x$ contain information about such a $D$-term. Our next step is to study contributions from different parts of the GPDs involved in the sum rule (25).

"Secondary" sum rule. One can easily see from Eq. (26) that

$$
\int_{-1}^{1} \frac{H_{+}(x, \xi)}{x} d x=0
$$

for any $\xi$, including $\xi=0$. Since the integrand is an even function of $x$, the vanishing of this integral means that zero is also obtained if we change the integration limits to $(0,1)$. Thus, $H_{+}(x, \xi)$ should be negative in some part of the central region, and this negative contribution should exactly compensate the contribution from the regions, where $H_{+}(x, \xi)$ is positive. In other words, on the $(0,1)$ interval, $H_{+}(x, \xi) / x$ has the same property as a "plus distribution" with respect to $x$. Note, however, that for finite $\xi$, the function $H_{+}(x, \xi) / x$ is pretty regular for all $x$ values (see Fig. 4).

The negative $\delta(x)$ function appears only in the $\xi=0$ limit, i.e.

$$
\frac{H_{+}(x, 0)}{x}=\frac{f(x)}{x}-\delta(x) \int_{-1}^{1} \frac{f(y)}{y} d y \equiv\left[\frac{f(x)}{x}\right]_{+} .
$$

For the integral involving the border function $H_{+}(x, x)$, we get

$$
\int_{-1}^{1} \frac{H_{+}(x, x)}{x} d x=\int_{-1}^{1} d x \int_{\Omega} d \beta d \alpha f(\beta, \alpha)\left\{\delta[x(1-\alpha)-\beta]-\frac{\delta(x)}{1-\alpha}\right\} .
$$

The integrals coming from the two delta-functions cancel each other, and we have

$$
\int_{-1}^{1} \frac{H_{+}(x, x)}{x} d x=0
$$

just like for $H_{+}(x, \xi) / x$. Unlike $H_{+}(x, \xi)$, however, the combination $H_{+}(x, x) / x$ explicitly contains the $\delta(x)$ subtraction term, i.e. it is a genuine "plus distribution" with respect to $x$, namely, $H_{+}(x, x) / x=[H(x, x) / x]_{+}$.

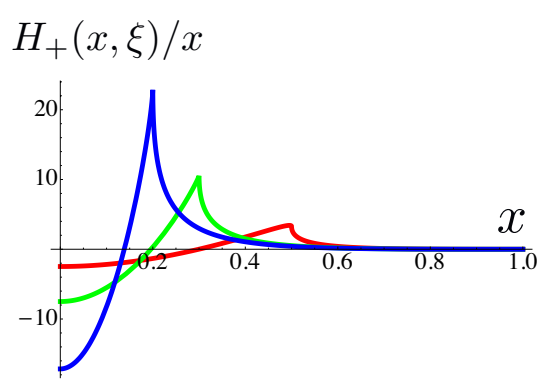

Fig. 4. Function $H_{+}(x, \xi) / x$ in the $N=1$ profile model for $\xi=0.2,0.3,0.5$ and positive $x$. 
Let us now turn to the $D$-parts. First, we have

$$
\int_{-1}^{1} \frac{H_{D}(x, \xi)}{x} d x=\int_{-1}^{1} \frac{D(\alpha)}{\alpha} d \alpha
$$

for any fixed $\xi$, including $\xi=0$. This result may be obtained by integrating over $x$ the $\delta(x-\xi \alpha)$ factor in the integral representation (27). For the integral involving the border function, we use Eq. (29), which gives

$$
\int_{-1}^{1} \frac{H_{D}(x, x)}{x} d x=\int_{-1}^{1} \frac{D(\alpha)}{\alpha(1-\alpha)} d \alpha .
$$

As a result,

$$
\int_{-1}^{1} \frac{H_{D}(x, x)}{x} d x-\int_{-1}^{1} \frac{H_{D}(x, 0)}{x} d x=\int_{-1}^{1} \frac{D(\alpha)}{1-\alpha} d \alpha .
$$

Adding zero contributions from the "plus" parts, one obtains the sum rule (25).

An essential point is that both $H_{D}(x, 0) / x$ and $H_{D}(x, x) / x$ are proportional to the $\delta(x)$-function, with the coefficients given by integrals of the $D$-term function $D(\alpha)$. In this sense, $H(x, 0) / x$ and $H(x, x) / x$ "know" about the $D$-term.

A simple consequence is that all $x^{j}$ moments of $H_{D}(x, 0)$ and $H_{D}(x, x)$ with $j \geq 0$ vanish, and one cannot get the $D$-part of the sum rule (25) by an analytic continuation of the $x^{j}$ moments of $H_{D}(x, 0)$ and $H_{D}(x, x)$ to $j=-1$, i.e., by using the procedure of Refs. $[18,21,22]$. In fact, $x^{j}$ moments of $H_{D}(x, 0)$ and $H_{D}(x, x)$ are proportional to the Kronecker delta function $\delta_{j,-1}$, a non-analytic function of $j$.

Need for renormalization. Since $H(x, 0) / x$ is given by integrating the DD $f(\beta, \alpha)$ over $\alpha$ along vertical lines $\beta=x$, a subsequent integration over all $x$ gives DD $f(\beta, \alpha)$ integrated over the whole rhombus:

$$
\int_{-1}^{1} \frac{H(x, 0)}{x} d x=\int_{\Omega} f(\beta, \alpha) d \beta d \alpha=\int_{-1}^{1} \frac{D(\alpha)}{\alpha} d \alpha .
$$

On the last step, we used that the $\beta$-integral of $f(\beta, \alpha)$ formally gives $D(\alpha) / \alpha$. However, if $f(\beta, \alpha) \sim 1 / \beta^{1+a}$, being even in $\beta$, one needs a regularization for the $\beta$-integral. The "DD+D" separation (1), as we have seen, provides such a regularization. It works like a renormalization: the divergent integral formally giving the $D$-term is subtracted from the "bare" DD, and substituted by a finite "observable" function $D(\alpha) / \alpha$. In a similar way, we can treat the second integral:

$$
\int_{-1}^{1} \frac{H(x, x)}{x} d x=\int_{\Omega} \frac{f(\beta, \alpha)}{1-\alpha} d \beta d \alpha=\int_{-1}^{1} \frac{D(\alpha)}{\alpha(1-\alpha)} d \alpha .
$$

Again, the last step requires a subtraction of the infinite part of the $\beta$-integral.

The advantage of using the " $\mathrm{DD}_{+}+\mathrm{D}$ " separation as a renormalization prescription is that it is applied directly to the DD. Hence, it is universal, and may be used for other integrals involving $f(\beta, \alpha)$. 


\section{Analytic Regularization}

Mellin moments. In Refs. [18, 21, 22] it was proposed to renormalize the $\beta$-integral in Eq. (37) by analytic regularization. Namely, it is assumed that the positive Mellin moments (or conformal moments, see, e.g., Ref. [36])

$$
\Phi(j) \equiv \int_{-1}^{1} x^{j}[H(x, x)-H(x, 0)] d x
$$

can be analytically continued to the point $j=-1$. The result of such a procedure is equivalent to analytic regularization of the $x$-integral. However, the assumed analyticity properties of $\Phi(j)$ may be violated by singular or "invisible" terms (cf. Ref. [18]) in the integrand of Eq. (39). For example, a $x \delta(x)$ term gives a non-analytic $\delta_{j,-1}$ contribution into $\Phi(j)$. Such terms explicitly emerge as a result of subtractions in the dispersion relation (12), so one may wish to develop a less restrictive approach to the renormalization problem. In this connection, we would like to stress that the derivation of the sum rule (25) given above was based merely on separation (7) of the DDs into the "plus" part and the $D$-term. No assumptions about smoothness were made.

Comparison of the "plus" prescription and analytic regularization. Analytic regularization works as follows. If we need to integrate a function like $\lambda(x) / x^{a+1}$ with $\lambda(x)$ being finite and nonzero for $x=0$, we subtract from $\lambda(x)$ as many terms of its Taylor expansion as needed to remove the divergence

$$
\begin{aligned}
\int_{(0)}^{y} \frac{\lambda(x)}{x^{a+1}} d x= & \int_{0}^{y} d x \frac{\lambda(x)-\lambda(0)-x \lambda^{\prime}(0)-\ldots}{x^{a+1}} \\
& +\lambda(0) \int_{(0)}^{y} \frac{d x}{x^{a+1}}+\lambda^{\prime}(0) \int_{(0)}^{y} \frac{d x}{x^{a}}+\ldots,
\end{aligned}
$$

and then treat the compensating integrals of $x^{n} / x^{a+1}$ as convergent, substituting them by $y^{n-a} /(n-a)$. So, let us consider a DD which is nonzero for positive $\beta$ only and has the form $f(\beta, \alpha)=\lambda(\beta, \alpha) / \beta^{a+1} \theta(\beta+|\alpha| \leq 1) \theta(\beta \geq 0)$ with $a<1$. Then the analytic regularization of its integral with some reference function $\Phi(\beta)$ is defined by

$$
\int_{(0)}^{1-|\alpha|} \frac{\Phi(\beta) \lambda(\beta, \alpha)}{\beta^{a+1}} d \beta=\int_{0}^{1-|\alpha|} \frac{\Phi(\beta) \lambda(\beta, \alpha)-\Phi(0) \lambda(0, \alpha)}{\beta^{a+1}} d \beta-\frac{\Phi(0) \lambda(0, \alpha)}{a(1-|\alpha|)^{a}},
$$

which may be rewritten as

$$
\begin{array}{r}
\int_{(0)}^{1-|\alpha|} \Phi(\beta) \frac{\lambda(\beta, \alpha)}{\beta^{a+1}} d \beta=\int_{0}^{1-|\alpha|}[\Phi(\beta)-\Phi(0)] \frac{\lambda(\beta, \alpha)}{\beta^{a+1}} d \beta \\
+\Phi(0)\left[\int_{0}^{1-|\alpha|} \frac{\lambda(\beta, \alpha)-\lambda(0, \alpha)}{\beta^{a+1}} d \beta-\frac{\lambda(0, \alpha)}{a(1-|\alpha|)^{a}}\right] .
\end{array}
$$


Now, the first contribution on the r.h.s. is generated by the "plus" part of the DD, while the second one corresponds to a $D$-term. After adding the $\beta<0$ part of the $\mathrm{DD}$, the $D$-term $\mathcal{D}(\alpha) / \alpha$ corresponding to the analytic regularization is given by

$$
\frac{\mathcal{D}(\alpha)}{\alpha}=2\left[\int_{0}^{1-|\alpha|} \frac{\lambda(\beta, \alpha)-\lambda(0, \alpha)}{\beta^{a+1}} d \beta-\frac{\lambda(0, \alpha)}{a(1-|\alpha|)^{a}}\right] .
$$

Thus, the analytic regularization prescription unambiguously fixes the $D$ term, and in this sense it may be called the "analytic renormalization". In particular, for the $N=1$ model, we have $\lambda(\beta, \alpha)=\frac{3}{4}\left[(1-\beta)^{2}-\alpha^{2}\right]$, and, hence,

$$
\frac{\mathcal{D}(\alpha)}{\alpha}=\frac{3}{2}\left[\frac{(1-|\alpha|)^{2-a}}{2-a}-2 \frac{(1-|\alpha|)^{1-a}}{1-a}-\frac{1-\alpha^{2}}{a(1-|\alpha|)^{a}}\right] \text {. }
$$

In Fig. 5, we compare this result (for $a=0.5$ ) with the result obtained in the model with implanted Regge behavior based on single subtraction in the dispersion relation (12) and $T_{0}=0$.

One can always add in the latter the $D_{0}$ term coming from the $T_{0}$ constant in (12). To analytically compare the $D$-term of the $T_{0}=0$ model of the previous section with that obtained via analytic renormalization, we take $\lambda(\beta, \alpha)=\varphi(\beta) h(\beta, \alpha)$ (where $\varphi(\beta)=(1-\beta)^{3}$ in our model for $f(\beta)$ ). Then it is possible to show that

$$
D(\alpha)-\mathcal{D}(\alpha)=2 \alpha h(0, \alpha)\left[\frac{1}{a}+\int_{0}^{1} \frac{d \beta}{\beta^{a+1}}\left(1-\frac{\varphi(\beta)}{(1-\beta)^{2}}\right)\right] .
$$

The expression in the square brackets here is just a number, call it $t_{0}(a)$. As expected, the two prescriptions differ by a term proportional to $\alpha h(0, \alpha)$, i.e., they can be made identical by adjusting the value of $T_{0}$ to be equal to $-t_{0}(a)$.

This means that the analytic regularization is just a particular case of the model involving adjustable subtraction constant $T_{0}$. Still, the statement, that $x^{j}$ moments of $H(x, \xi)$ are analytic functions of $j$, does not explicitly mention fixing any subtraction constants: it may create an impression that there are no ambiguities in the subtraction of the $\beta=0$ singularity. However, the analyticity assumption was

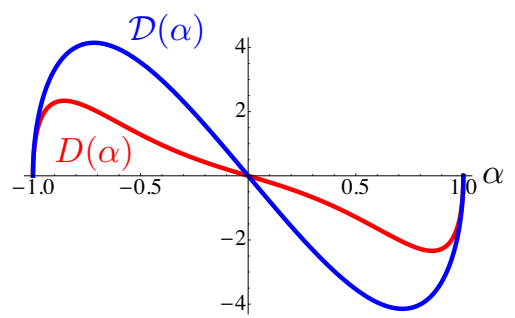

Fig. 5. The $D$-terms in the model with $N=1$ profile and $a=0.5: \mathcal{D}(\alpha)$ was obtained using analytic regularization, and $D(\alpha)$ was obtained for $T_{0}=0$ in the model of the previous section. 
not shown so far to be a consequence of general principles of quantum field theory. Moreover, ${ }^{37}$ it is not satisfied in the nonlocal chiral soliton model.

Summary on sum rules. Thus, our calculation supports the $\xi=0$ sum rule (25) suggested in Ref.[17]. Since $H(x, 0) / x$ and $H(x, x) / x$ are even functions of $x$, the $\xi=0$ sum rule may be written through an integral from 0 to 1 , and its $1 / x$ singularity is at the end-point of the integration region, which means that the $P$ prescription cannot regulate it. Because of this fact alone, the sum rule (25) cannot be a straightforward consequence of the generic sum rule (24). In our derivation, the finite expressions were obtained for all terms involved in the sum rule. We made no assumptions about analyticity of the Mellin moments of GPDs. Basically, we have obtained GPD sum rules as a consequence of the polynomiality of GPDs that follows from Lorentz invariance and is encoded in the DD representation. The analyticity is a much stronger restriction. One may try to find out whether it can be tested experimentally and it is also worth trying to check if it is valid in QCD.

\section{Conclusions}

We discussed models for GPDs based on the factorized DD Ansatz (FDDA) within the "single-DD" formulation. The main difficulty in the implementation of such a construction is the necessity to deal with projection onto a more singular function $f(\beta) / \beta$ (rather than just onto $f(\beta)$ ) in the forward limit. This leads to two problems. First, one encounters non-integrable singularities for $\beta=0$ in the integrals producing GPDs in the central region $|x|<|\xi|$. The difficulty is exaggerated by necessity to consider forward distributions $f(\beta)$ that have a singular $\beta^{-a}$ Regge behavior at small $\beta$. Second, if there are no factors suppressing the $\beta \sim 0$ region for the integration line corresponding to $x=\xi$, the combined $1 / \beta^{1+a}$ singularity leads to a singular $(x-\xi)^{-a}$ behavior for GPDs in the outer region $x>\xi$ near the border point $x=\xi$. Such a behavior was found in the model of Ref. [34].

In our analysis, we found that the model of Ref. [34] gives the single-DD-type representation for the model GPD, and thus above reasoning is applicable to it. But we argued, that a proper softening of the hadron-quark vertices produces a profile function $h_{N}(\beta, \alpha)$ that results, for $x=\xi$, in the $\mathcal{O}\left(\beta^{N}\right)$ suppression factor securing a finite value of the $\operatorname{GPD} H(x, \xi)$ at the border point.

However, the profile factor has no impact on the combined $1 / \beta^{1+a}$ singularity on the $\beta=0$ line inside the support rhombus, which one faces when calculating GPDs in the $|x|<|\xi|$ region. The advantage of the model of Ref. [34] is that it implants the Regge behavior through a subtracted dispersion relation for the hadron-quark scattering amplitude. We found that the subtraction provides the regularization necessary for the calculation of GPDs in the central region, and illustrated the behavior of resulting GPDs in models with $N=1$ and $N=2$ profiles.

We also observed that this model produces a $D$-term contribution, despite the fact that it uses only the forward distribution as an input. This $D$-term contribution 
appears because the subtraction generated by the dispersion relation differs from the subtraction that converts the original DD into a "plus" distribution $[f(\beta, \alpha)]_{+}$. The latter, by definition, cannot generate a $D$-term. We have shown that the GPD $H_{+}(x, \xi)$ generated by the $[f(\beta, \alpha)]_{+}$part of the original DD (i.e., $\operatorname{GPD} H(x, \xi)$ with the $D$-term contribution $D(x / \xi)$ subtracted) has a remarkable property that the integral of $H_{+}(x, \xi) / x$ over positive values $0 \leq x \leq 1$ vanishes. As a result, $H_{+}(x, \xi)$ must be negative in some part of the central region, a feature that is absent in previous FDDA models based on two-DD formulation.

Within the single-DD formalism, it is very natural to separate the relevant DD $f(\beta, \alpha)$ into the "plus" part $[f(\beta, \alpha)]_{+}$and the $D$-term. This separation can be used to rederive the GPD sum rule related to the dispersion relation for the real part of the DVCS amplitude. ${ }^{15}$ Here, we demonstrated that it also can be used for a derivation of another sum rule proposed as the $\xi \rightarrow 0$ limit of that generic sum rule. Our derivation shows that in the "secondary" sum rule, instead of the principal-value prescription, one should use a "plus"-type prescription. The "plus" prescription, in fact, is automatically generated by the separation of DDs into the "plus" part and the $D$-term.

Summarizing, we illustrated the basic tools that can be used in building realistic GPD models based on the factorized DD Ansatz within the single-DD formalism. Future developments in this direction should include the extension of the presented methods onto the cases with $a>1$ Regge behavior, which would require an extra subtraction in the dispersion relation, and building models for nucleons and other targets with a non-zero spin.

\section{Acknowledgments}

I thank H. Moutarde, D. Müller, K.M. Semenov-Tian-Shansky, A.P. Szczepaniak and O.V. Teryaev for stimulating discussions.

This work is supported by Jefferson Science Associates, LLC under U.S. DOE Contract No. DE-AC05-06OR23177.

\section{References}

1. D. Muller, D. Robaschik, B. Geyer, F. M. Dittes and J. Horejsi, Fortsch. Phys. 42, 101 (1994).

2. X. D. Ji, Phys. Rev. Lett. 78, 610 (1997).

3. X. D. Ji, Phys. Rev. D 55, 7114 (1997).

4. A. V. Radyushkin, Phys. Lett. B 380, 417 (1996).

5. A. V. Radyushkin, Phys. Lett. B 385, 333 (1996).

6. A. V. Radyushkin, Phys. Rev. D 56, 5524 (1997).

7. J. C. Collins, L. Frankfurt and M. Strikman, Phys. Rev. D 56, 2982 (1997).

8. X.-D. Ji, J. Phys. G G 24, 1181 (1998).

9. K. Goeke, M. V. Polyakov and M. Vanderhaeghen, Prog. Part. Nucl. Phys. 47, 401 (2001).

10. M. Diehl, Phys. Rept. 388, 41 (2003).

11. A. V. Belitsky and A. V. Radyushkin, Phys. Rept. 418, 1 (2005). 
12. S. Boffi and B. Pasquini, Riv. Nuovo Cim. 30, 387 (2007).

13. M. V. Polyakov and C. Weiss, Phys. Rev. D 60, 114017 (1999).

14. A. V. Radyushkin, "Basics of generalized parton distributions", these Proceedings.

15. A. V. Radyushkin, Phys. Rev. D 83, 076006 (2011).

16. O. V. Teryaev, hep-ph/0510031 (2005).

17. I. V. Anikin and O. V. Teryaev, Phys. Rev. D 76, 056007 (2007).

18. K. Kumericki, D. Mueller and K. Passek-Kumericki, Nucl. Phys. B 794, 244 (2008).

19. M. Diehl and D. Y. Ivanov, Eur. Phys. J. C 52, 919 (2007).

20. O. V. Teryaev, PoS DIS 2010, 250 (2010).

21. K. Kumericki, D. Mueller and K. Passek-Kumericki, Eur. Phys. J. C 58, 193 (2008).

22. M. V. Polyakov and K. M. Semenov-Tian-Shansky, Eur. Phys. J. A 40, 181 (2009).

23. X.-D. Ji, W. Melnitchouk and X. Song, Phys. Rev. D 56, 5511 (1997).

24. V. Y. Petrov, P. V. Pobylitsa, M. V. Polyakov, I. Bornig, K. Goeke and C. Weiss, Phys. Rev. D 57, 4325 (1998).

25. M. Diehl, T. Feldmann, R. Jakob and P. Kroll, Eur. Phys. J. C 8, 409 (1999).

26. A. V. Radyushkin, Phys. Rev. D 59, 014030 (1999).

27. L. Mankiewicz, G. Piller and T. Weigl, Eur. Phys. J. C 5, 119 (1998).

28. I. V. Musatov and A. V. Radyushkin, Phys. Rev. D 61, 074027 (2000).

29. A. V. Belitsky, D. Mueller, A. Kirchner and A. Schafer, Phys. Rev. D 64, 116002 (2001).

30. A. V. Radyushkin, Phys. Lett. B 449, 81 (1999).

31. A. Mukherjee, I. V. Musatov, H. C. Pauli and A. V. Radyushkin, Phys. Rev. D 67, 073014 (2003).

32. D. S. Hwang and D. Mueller, Phys. Lett. B 660, 350 (2008).

33. A. Efremov and A. Radyushkin, Mod. Phys. Lett. A 24, 2803 (2009).

34. A. P. Szczepaniak, J. T. Londergan and F. J. Llanes-Estrada, Acta Phys. Polon. B 40, 2193 (2009).

35. M. V. Polyakov, Phys. Lett. B 659, 542 (2008).

36. K. Kumericki and D. Mueller, Nucl. Phys. B 841, 1 (2010).

37. K. M. Semenov-Tian-Shansky, Eur. Phys. J. A 36, 303 (2008). 